\title{
Software Receiver Design for Galileo Signals
}

\author{
R. de Lezaeta and R.M. Rodríguez-Osorio
}

\begin{abstract}
The article presents a new concept of softwarebased GNSS receivers because of its advantages in flexibility and possibility of reconfiguration. The aim is to develop a receiver for navigation signals of the Galileo system testbed, the Giove-A and Giove-B. The receiver must be able to acquire, track and demodulate signals "BOC" system. For this test different techniques, both in the acquisition and follow-up. Also developed a configurable signal generator for testing and a graphical user interface to manage the receiver easily. The results show that the signals can be acquired successfully with a front-end narrowband GPS and conventional algorithms. Similarly, you can track and demodulate the data channel signal (E1B) using the BOCPRN configuration.
\end{abstract}

Keywords-Galileo, GNSS receivers, GAEDUNAV.

\section{INTRODUCCIÓN}

$\mathrm{L}$ A navegación global por satélite a través de los sistemas GNSS (Global Navigation Satellite Systems) es actualmente uno de los campos de mayor actividad en la industria espacial. Uno de los principales motivos de su auge se debe al gran número de aplicaciones que pueden desarrollarse a partir de los datos de posición y navegación obtenidos en un receptor. El uso de los sistemas GNSS no sólo se limita a los usuarios domésticos, sino que también se usan para ofrecer servicios marítimos, aeronáuticos, de emergencias o de transporte [1].

En la actualidad, el sistema empleado masivamente es el americano GPS. En el 2013, se desplegará la iniciativa europea en el campo de navegación global por satélite, conocida como Galileo. Como paso previo al despliegue final del sistema, en 2005 se lanzó el primer satélite de la constelación Galileo para la realización de pruebas y la validación del sistema (Giove-A). Más recientemente, en abril de 2008 se lanzó el Giove-B, sucesor del satélite Giove-A, y en 2010 está planeado el lanzamiento de los satélites IOV (In Orbit Validation) que serán casi iguales a los usados en la constelación final.

La tecnología de receptores software se basa en la digitalización tan cerca de la antena como sea posible para que

Los autores agradecen a la Cátedra ISDEFE de la ETSIT-UPM y a la Comisión Interministerial de Ciencia y Tecnología CICYT bajo el proyecto TEC2008-06736/TEC por la financiación y apoyo al proyecto.

R. de Lezaeta ha terminado recientemente sus estudios en la Escuela de Ingeniería de Telecomunicación de la Universidad Politécnica de Madrid y planea trabajar en el sector GNSS en el sistema Galileo ó GPS.

R. M. Rodríguez-Osorio, Profesor titular en el Departamento de Señales, Sistema y Radiocomunicaciones de la E.T.S.I. de telecomunicación de la Universidad Politécnica de Madrid, Avenida Complutense $\mathrm{n}^{\circ} 30$, "Ciudad Universitaria". 28040 - Madrid (España) (correos e.: rdlezaeta@gmail.com; ramon(a)gr.ssr.upm.es). el procesado (conversión de frecuencias, filtrado y demodulación) sea realizado totalmente en el dominio digital. Las ventajas de una implementación basada en software radio se traducen en una mayor flexibilidad, posibilidad de reconfiguración y menor consumo y tamaño. Además, es una excelente forma para probar nuevos diseños $\mathbf{y}$ prototipos, como paso previo a su implementación en hardware.

Los principales motivos para la realización de este proyecto se basaron en la contribución al avance y la expansión del conocimiento del sistema Galileo. En esta línea se buscó la simplificación y la implementación de los equipos del segmento usuario (receptores) desde un nuevo punto de vista (software), para obtener estructuras modificables y funcionales que pudiesen servir como pie a nuevos desarrollos y de ayuda a la docencia, además de comprobar y demostrar las ventajas de la tecnología software en este tipo de equipos.

\section{TEORÍA}

Un receptor software para sistemas de navegación por satélite requiere varios módulos hardware además de los correspondientes de software. El procesado de señales se realiza enteramente en un PC con un programa organizado en distintas etapas, mientras que la captación, acondicionamiento y muestreo de dichas señales se lleva a cabo con un dispositivo integrado de RF. El receptor (front-end) capta las emisiones del satélite a través de una antena omnidireccional, las procesa y da a su salida muestras en FI o en banda base, dependiendo de la versión. Son estas muestras las que posteriormente se entregan al receptor software. Es por ello que un receptor como el diseñado puede integrarse con cualquier front-end que proporcione muestras a una FI determinada. El esquema se puede ver en la Figura 1.

\section{A. Diagrama de bloques del receptor software}

La estructura general del receptor software se puede dividir en tres grandes áreas: Adquisición, Tracking y procesado de información (Figura 2). El objetivo final, como sabemos, es obtener la posición del receptor, pero para ello es necesario extraer previamente los datos de navegación. Con esta apreciación podemos dividir el receptor en dos etapas: por un lado, la adquisición y el tracking que se encargan de extraer los bits de navegación y por el otro todo lo relativo al cálculo de la posición a partir de dichos bits. La parte de adquisición y tracking trabaja para inicializar y seguir las variaciones en frecuencia y fase de la señal. Esto es muy importante pues de lo contrario no se podrían demodular los bits de navegación. 


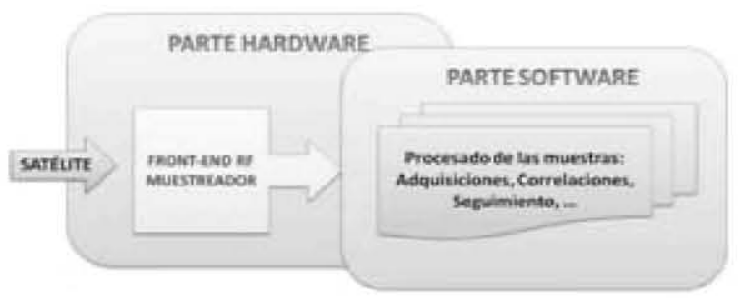

Figura 1. Esquema hardware y software del receptor

El diseño de receptor se realizó en Matlab de acuerdo a dos características principales: la primera, que las etapas estuviesen claramente diferenciadas y fueran independientes, y la segunda, que el funcionamiento de sus módulos fuese fácilmente modificable.

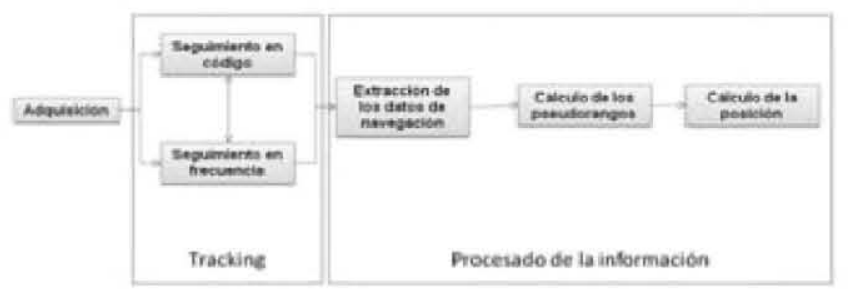

Figura 2. Estructura del receptor software

Para la primera característica se buscó que cada etapa, al finalizar, generase un fichero de resultados que se pudiese guardar para ser utilizado por la etapa posterior tantas veces como se desee sin necesidad de ejecutar otra vez la anterior. Estos ficheros de resultados diferencian claramente dónde acaba un módulo y dónde empieza otro.

Para la segunda característica se cuenta con un fichero que contiene y controla todos los parámetros del receptor. Su función es inicializarlos y guardarlos. Los parámetros se pueden modificar bien directamente en el fichero ó corriendo una GUI dedicada. Como resultado de su ejecución devuelve una estructura donde se encuentran todos los parámetros.

\section{B. Estructura de la señal}

Las señales utilizadas en el futuro sistema Galileo forman parte de las denominadas Binary Offset Carrier $(m, n)$ (ó $\mathrm{BOC}(\mathrm{m}, \mathrm{n}))$. En particular la modulación utilizada en la señal de servicio abierto (OS) es la MBOC (Multiplex. BOC) y está formada por una $\operatorname{BOC}(1,1)$ y una $B O C(6,1)$. Sus ventajas frente a las señales de GPS usuales (BPSK) residen en una mayor robustez frente al efecto del multitrayecto y un mayor rechazo de interferencias de banda estrecha. Aunque también permite una compartición adecuada del espectro en la banda L1 (1159 - 1610 $\mathrm{MHz}$ ).

La señal E1 (OS) está compuesta por tres canales A-B-C [2]. El canal B (eE1-B(t)) corresponde a la señal con los datos de navegación y el C (eE1-C(t)) al canal piloto, mientras que la señal del canal A (eEl-A(t)) es de uso gubernamental y está encriptada. Los códigos primarios se generan con una estructura LFSR (Linear Feedback Shift Register) cuyas semillas y taps se encuentran en el ICD (Interface Control
Document) de Giove [2]. La combinación de los tres canales se hace con un esquema CASM (Coherent Adaptative Subcarrier Modulation) para mantener constante la envolvente en potencia, es decir, para que la potencia transmitida no varie con el tiempo.

\section{Módulo de adquisición}

El propósito de la función de adquisición en un receptor de navegación por satélite es identificar todos los satélites visibles. Si un satélite es efectivamente visible, el proceso de adquisición debe determinar su frecuencia y su desfasaje en código (fase del código) para su posterior procesado $\mathrm{y}$ utilización en el cálculo de la solución de navegación.

Para buscar la frecuencia de la señal y el desplazamiento en código, el algoritmo de adquisición debe ir realizando pruebas con parejas de frecuencia y offset de código. Para ello existen 3 rutinas generales que se diferencian en complejidad y duración. Éstas son: Serial search, Parallel frequency space search y Parallel code phase search. Se decidió usar la búsqueda en paralelo del offset de código (Parallel code phase Search) debido a que minimizaba el número de iteraciones. Este método es el más complejo y conlleva el uso de FFT's e IFFT's para realizar las correlaciones en el dominio de la frecuencia. La ventaja es que comprueba todas las fases de código a la vez (4092 para E1B y 8184 para E1C) en cada salto de frecuencia. Los saltos se fijaron en $62.5 \mathrm{~Hz}$ porque permitían una solución razonable entre resolución y carga computacional. El margen de búsqueda se fijó en $14 \mathrm{KHz}$ alrededor de la portadora, un caso peor para un sistema GNSS. El diagrama del módulo de adquisición se muestra en la Figura 3.

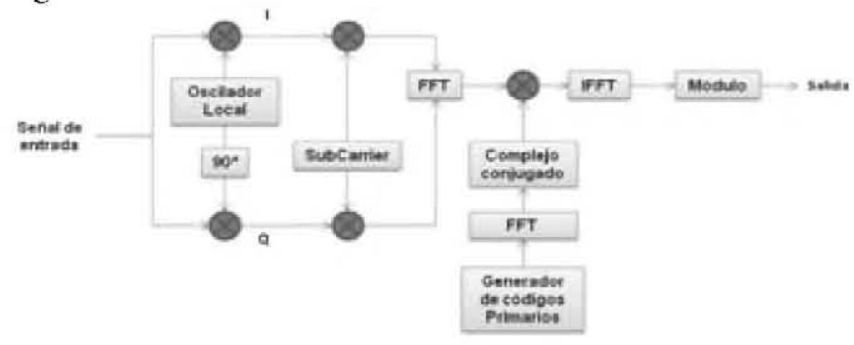

Figura 3. Diagrama del módulo de adquisición: Parallel code phase search

Posteriormente para afinar el valor de la frecuencia se utilizan 10 periodos de señal (para cada código) en un procesado con FFT's, multiplicando por $8 \mathrm{el}$ número de puntos para el cálculo de la transformada. Este procedimiento se denomina adquisición gruesa ó "coarse acquisition" y da una estimación inicial aproximada.

\section{Módulo de seguimiento}

El sistema de seguimiento (tracking) está a continuación del módulo de adquisición y tiene básicamente dos funciones. La primera es realizar un seguimiento (Fine acquisition) de la señal adquirida en frecuencia y en código para no perderla (pues habría que volver a adquirirla). No olvidemos que al estar moviéndose el satélite, los valores de frecuencia y offset de código cambian con el tiempo.

En segundo lugar, mientras se hace el seguimiento, el sistema 
debe demodular los datos de navegación del satélite específico que se está siguiendo. Para demodular, el procedimiento básico es multiplicar la señal recibida por una réplica de la portadora y luego por una réplica del código primario y la subportadora. Todas estas réplicas son estimadas por el algoritmo de seguimiento. El bloque está formado por un Bucle de Costas [3] que incluye un DLL para realizar el rastreo en frecuencia y código, respectivamente. Se usa un Bucle de Costas porque es insensible a cambios de fase de $180^{\circ}$, que se producen al menos una vez en cada periodo de código [4]. El discriminador utilizado para la señal E1B es el de arco tangente (1). Este discriminador es el más preciso para el Bucle de Costas, pero también es el que tarda más. Sin embargo, tiene la ventaja de que su salida es directamente el error de fase. Para el canal piloto (E1C) usamos el discriminador arco tangente extendido (2).

$$
\begin{aligned}
& Q_{\text {errer }}=\operatorname{arctg}\left(\frac{Q}{I}\right) \quad[Q] \\
& \varphi_{\text {frror }}=\operatorname{arctg} 2(Q, 1)[\because]
\end{aligned}
$$

Las razones de usar el algoritmo extendido para el piloto son que cuenta con el rango lineal de seguimiento más grande, ya que cubre enteramente el rango de $[-\pi, \pi]$ rads [5]. Esto permite la tolerancia de un error de fase relativamente grande sin pérdida de enganche. Además, tampoco necesita normalización lo que reduce la complejidad.

En lo que se refiere al DLL, se usa un esquema correlación de tres réplicas, una alineada con el código (prompt), otra adelantada (early) y otra atrasada (late). Al contrario que para GPS, la señal $\operatorname{BOC}(1,1)$ de Galileo presenta dos problemas extras: El doble de ancho de banda del lóbulo principal y los picos secundarios en la función de auto-correlación que pueden causar ambigüedades [6]. El problema de la ambigüedad es el más importante porque puede causar que el DLL se enganche en un punto erróneo e introduzca serios errores en la medida de los pseudorangos. Para resolver estos problemas se han estudiado varios algoritmos y al final se decidió utilizar el discriminador BOC-PRN (E+L) [6]. La arquitectura BOC-PRN (E+L) es una extensión de los métodos BOC-PRN que se basan en mezclar la señal de entrada (BOC) con las mismas réplicas del código primario (PRN) únicamente. Por tanto, se obvia el efecto de la modulación de la subportadora en las ramas early y late, mientras que en la rama prompt sí se elimina dicho efecto porque es necesario para poder determinar el Doppler de la portadora.

El error de fase en frecuencia y en código se realimenta igual que para GPS pero además es necesario corregir la subportadora generada localmente para la mezcla. Las ventajas que presenta son: que elimina los puntos ambiguos, tiene una pendiente relativamente alta en la función del discriminador y permite la reducción del efecto del multitrayecto debido al espaciamiento 'early/late' conFig. ble. La expresión del discriminador es:

$$
\mathrm{D}(\mathrm{x})=\left(\mathrm{I}_{\mathrm{E}}+\mathrm{I}_{\mathrm{L}}+\mathrm{Q}_{\mathrm{E}}+\mathrm{Q}_{\mathrm{L}}\right)_{F_{\mathrm{Z}}}
$$

Donde $\mathrm{K}$ es un factor que depende de la frecuencia de muestreo y la potencia de señal de manera que el error de fase esté acotado en un rango razonable. El esquema utilizado para el tracking es el de la Figura 4. Otros autores solucionan los problemas mencionados anteriormente usando un discriminador normalizado 'Early-Late' con un espaciamiento entre réplicas menor (0.2 chips) y un esquema BOC-BOC [7].

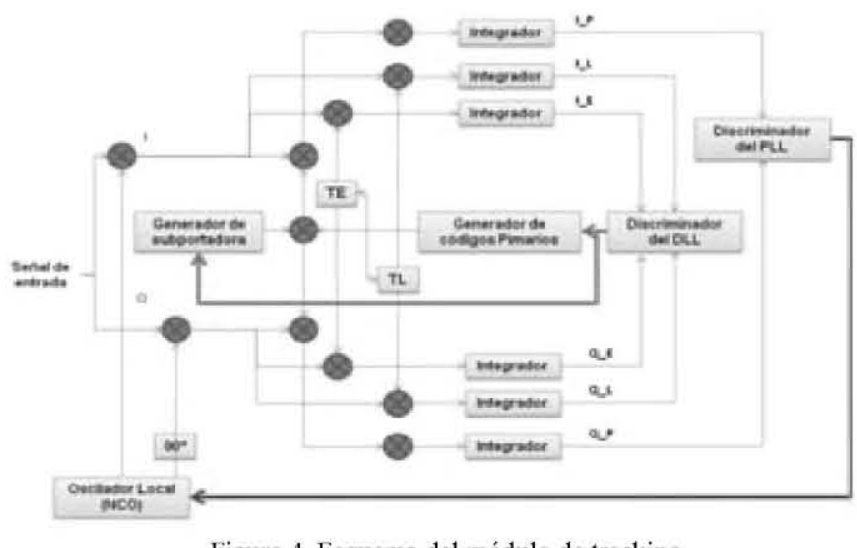

Figura 4. Esquema del módulo de tracking

\section{Simulaciones y PRUebas}

\section{A. Generador de señal}

Como paso previo al diseño del receptor se implementó un generador de señal que permitiese probar de manera sencilla el funcionamiento de los distintos módulos del receptor software. La importancia de esta herramienta radica en la config. bilidad que ofrece. Si se produjesen cambios en las señales del Giove-B o del Giove-A se podría adaptar el generador para cumplir las nuevas características. Casi todos los parámetros se pueden ajustar, de esta forma es posible trabajar con cada módulo de la manera que se desee. Para la generación de las señales se ha seguido el ICD de Galileo [2]. La implementación del generador se realizó en Matlab al igual que el receptor software.

El generador produce muestras de la envolvente compleja en frecuencia intermedia de la señal E1 con todos sus canales (A, B y C). Se pueden generar las dos señales E1 que se encuentran actualmente en funcionamiento con sus respectivas modulaciones: la emitida por el Giove-A y la emitida por el Giove-B. Las muestras se generan a una frecuencia de muestreo fija de $61.38 \mathrm{MHz}$. Este valor es 4 veces la frecuencia de la subportadora de señal del canal E1-A (15.345 MHz) y la razón de su elección es que da margen para realizar pruebas con distintas FI debido a que el ancho de banda total (E1) es de $32.736 \mathrm{MHz}$.

Las muestras se generan a una frecuencia intermedia modificable y es posible introducir la desviación Doppler de frecuencia, el offset de código para cada canal y la SNR deseadas para simular los datos tal y como los que provendrían de un frontend RF para GNSS. El espectro resultado de la generación puede verse en la Figura 5. (Giove-A) y en la Figura 6. (Giove-B). La frecuencia de muestreo utilizada es $61.38 \mathrm{MHz}$. 


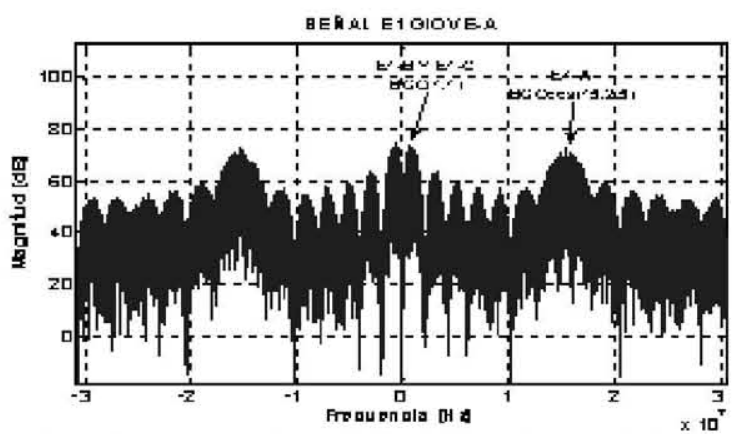

Figura 5. Espectro de la señal E1 del Giove-A en banda base

Para verificar que los espectros generados eran correctos comprobamos que los niveles relativos de los lóbulos fuesen los adecuados. En la Figura 6, tomando como referencia la componente de $1 \mathrm{MHz}$ con un nivel de $70 \mathrm{~dB}$ indicaremos entre paréntesis en valor (la diferencia) esperada y al lado la medida. Entre la referencia y el siguiente pico secundario tenemos $10.8 \mathrm{~dB}$ (10 dB), entre la referencia y la componente en $6 \mathrm{MHz}$ tenemos $11.4 \mathrm{~dB}(11 \mathrm{~dB})$ y entre la referencia y la componente E1-A tenemos $3.5 \mathrm{~dB}(4 \mathrm{~dB})$. Luego podemos confirmar que los espectros generados son muy parecidos a los reales.

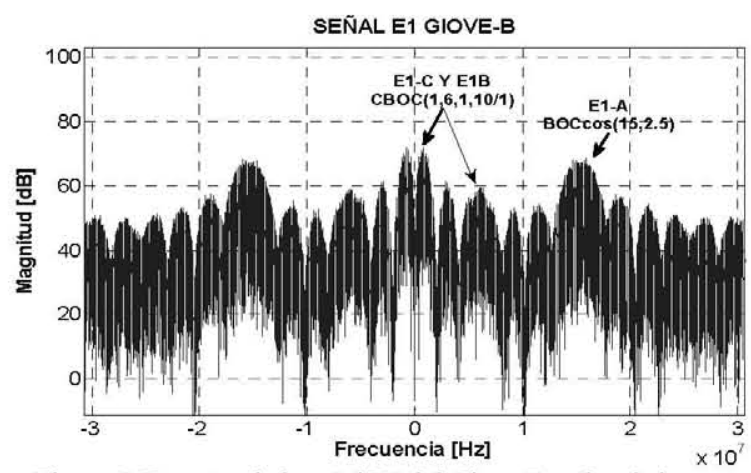

Figura 6. Espectro de la señal E1 del Giove-B en banda base

\section{B. Resultados de adquisición}

El funcionamiento del módulo de adquisición se comprobó tanto con señales generadas localmente como con señales provenientes de capturas. En la Figura 7 se aprecian los resultados de correlación en frecuencia y en código para la señal generada localmente. La frecuencia intermedia es $1 \mathrm{MHz}$, la frecuencia de muestreo es $12.276 \mathrm{MHz}$, el Doppler introducido es de $2000 \mathrm{~Hz}$, la SNR $=-30 \mathrm{~dB}$, los desfases de código en muestras es 1.135 para E1B y 4.657 para E1C.
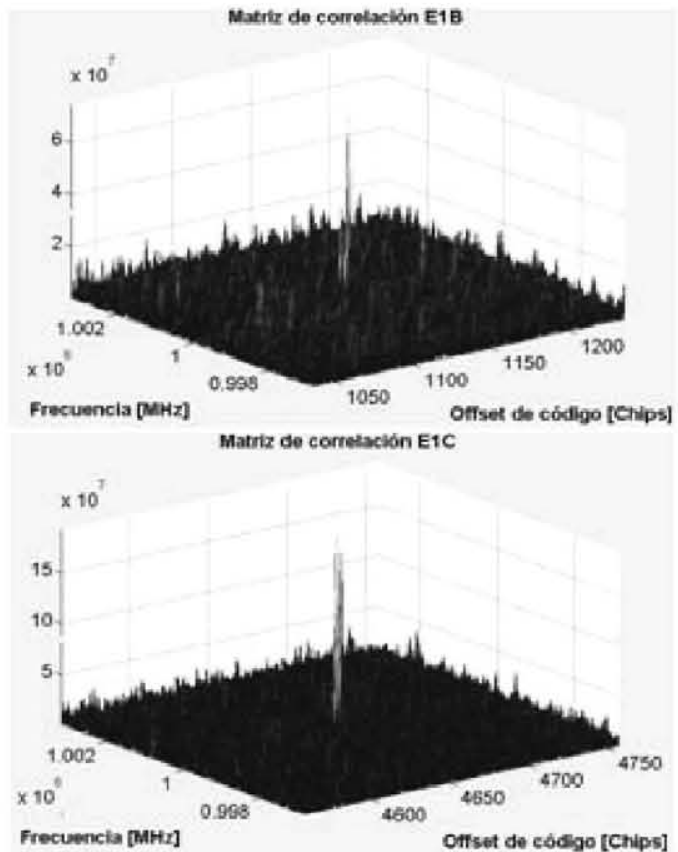

Figura 7. Matrices de correlación las señales E1B y E $1 \mathrm{C}$ (Giove-B). FI $=1$ $\mathrm{MHz}$, frec. Doppler $=2000 \mathrm{~Hz}, \mathrm{SNR}=-30 \mathrm{~dB}, \mathrm{E} 1 \mathrm{~B}$ codeOffset $=$ 1.135 chips y E1C code Offset $=4.657$ Chips.

Los resultados estimados en Doppler son $2020 \mathrm{~Hz}$ para E1B y $1992 \mathrm{~Hz}$ para E1C, y el offset de código estimado es de $1.135 \mathrm{y}$ 4.657 chips, respectivamente. La razón de que los valores de Doppler no coincidan con el introducido se debe a que el objetivo es hallar una primera estimación de la frecuencia de la portadora. La estimación precisa se hace en el siguiente módulo (tracking). Por otra parte, el que las estimaciones en ambas señales no sean exactamente iguales es consecuencia de que las FFT's usadas tienen distinto número de puntos dado que las longitudes de los periodos de código no son iguales.

Las pruebas con señales reales se llevaron a cabo con una señal capturada usando un receptor NordNav R30 en el Politécnico de Torino. Su frecuencia intermedia es $4.1304 \mathrm{MHz}$, su frecuencia de muestreo es $16.3676 \mathrm{MHz}$ y el formato de las muestras es un entero de 8 bits [4]. Los resultados se presentan en Figura 8 y la Figura 9.
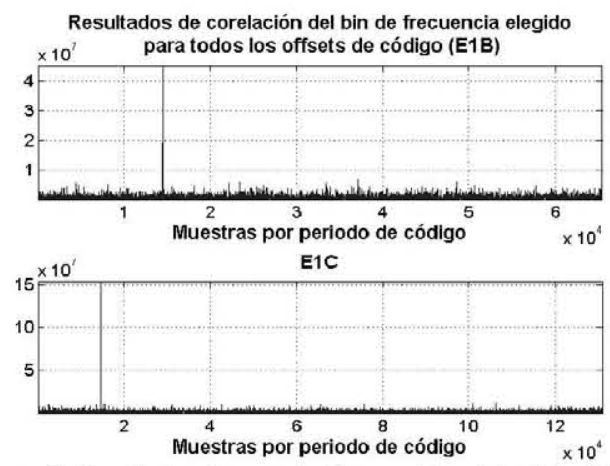

Figura 8. Resultados de correlación en el dominio del código 

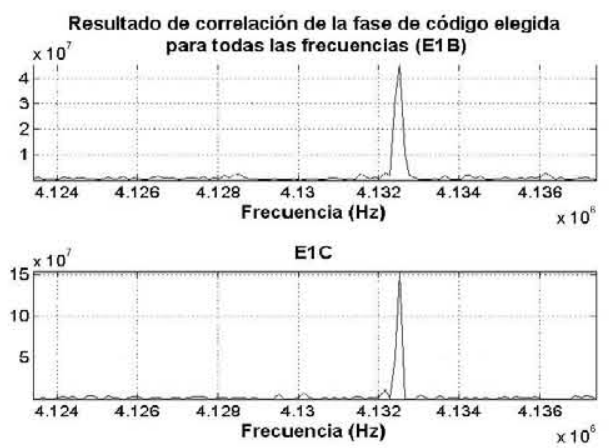

Figura 9. Resultados de correlación en el dominio de la frecuencia

El Doppler estimado en la señal E1B fue de $2.055 \mathrm{KHz}$ y en la señal E1C $2.052 \mathrm{KHz}$. Los offsets de código fueron $14.520 \mathrm{y}$ 14.519 muestras, respectivamente. Estos resultados se encuentran dentro de los valores que pueden alcanzarse en un pase. Por otra parte, es importante recalcar que las estimaciones son similares. Esto se debe a que los osciladores y relojes usados para generar la señal a bordo son los mismos, luego las perturbaciones en ambas señales serán muy parecidas.

Para observar el efecto del ruido, que en algunos casos enmascara la señal y no permite su detección, se probó el desempeño del algoritmo de adquisición con distintos valores de SNR. E1 Doppler introducido para cada SNR es constante e igual a $2000 \mathrm{~Hz}$ y la FI es igual a $1 \mathrm{MHz}$. En la Fig. 10 se representa la diferencia entre el valor estimado y el real en $\mathrm{Hz}$, y su variación en función de la SNR (dB) para ambos satélites. Los resultados finales se obtuvieron promediando 100 iteraciones del algoritmo para cada SNR. Cada iteración requería unos 8 minutos con lo cual para cada SNR de prueba eran necesarias unas 13 horas de simulación, lo que hace un total de unos 10 días por cada una de las gráficas. En condiciones normales el proceso de adquisición de una señal proveniente de alguna captura o de una generada localmente, es igual a unos 2 minutos en término medio. El menor mayor nivel de ruido soportable por el receptor fueron -30 dB's.

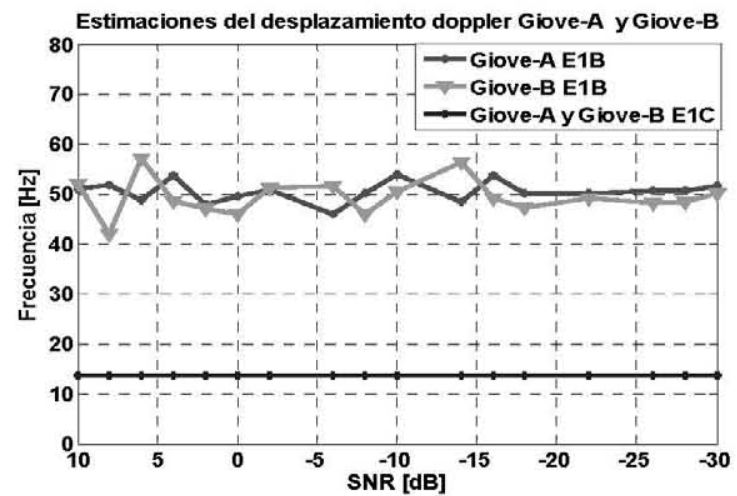

Figura 10. Desviaciones de la estimación Doppler para distintos valores de SNR

\section{Resultados de seguimiento}

Los resultados del seguimiento del canal de datos (E1B) con capturas de señal realizadas durante algún pase de satélite se pueden ver en las siguientes Fig. s. La Figura 11 muestra los bits de navegación extraídos de la señal de Giove-B proveniente de una captura realizada el 25-08-09 en Madrid con una elevación de $58.4^{\circ}$. La Figura 12 muestra los niveles de correlación durante el proceso, la traza verde identifica a la réplica centrada (prompt), la traza azul a la adelantada (early) y la roja a la atrasada (late). Como vemos, los resultados de la rama prompt obtienen un valor mayor y los de la rama late y la rama early se igualan, lo que implica que ambas están a la misma separación del offset correcto, es decir, el sistema está siguiendo adecuadamente a la señal.

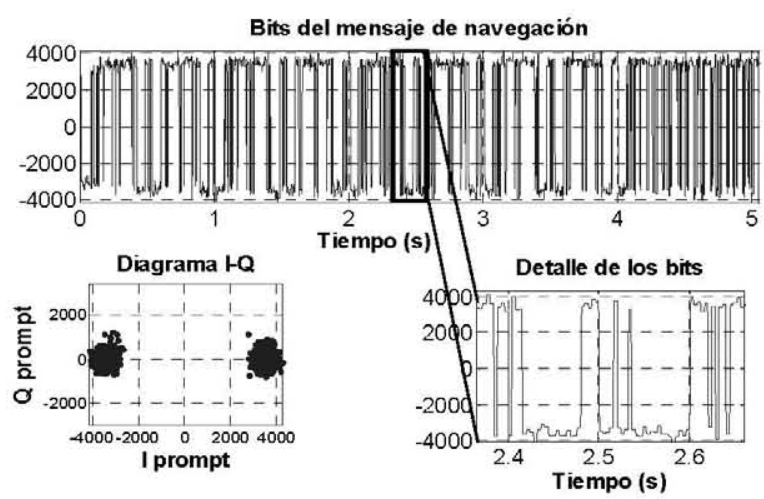

Figura 11. Bits de navegación extraídos de la captura de Giove-B realizada el 25-08-09 en Madrid con una elevación de $58.4^{\circ}$

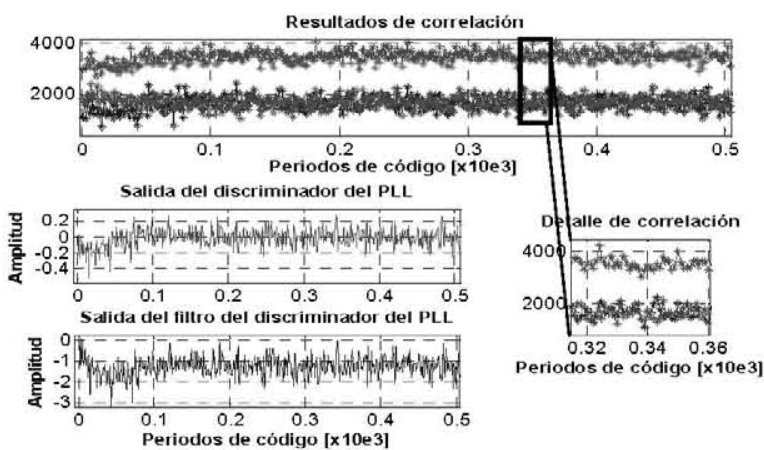

Figura 12. Resultados del DLL para la captura de Giove-B realizada el 25-0809 en Madrid con una elevación de $58.4^{\circ}$.

Igualmente, la Fig 13 y la Fig.14 muestran los mismos resultados para una captura de señal del Giove-A realizada el 30-06-09 en Madrid con una elevación de 52 . Los parámetros utilizados para el seguimiento de la señal se muestran en la tabla I. La duración del proceso de seguimiento depende de número de segundos que se quieran procesar, pero por ejemplo, para procesar 5 segundos de señal se requieren $1 \mathrm{~m} 51 \mathrm{~s}$. Los parámetros de la simulación se pueden ver en la Tabla I. 
TABLA I

PARAMETROS DE LA SIMULACIÓN

\begin{tabular}{|c|c|}
\hline Parámetro & E1B \\
\hline Disc. DLL & BOC-PRN (E-L) \\
\hline Disc. PLL & Atan \\
\hline Orden DLL/PLL & $2 \mathrm{do} / 2 \mathrm{do}$ \\
\hline Espaciamiento E-L & $0.6 \mathrm{chips}$ \\
\hline Tiempo de integración & $4 \mathrm{~ms}$ \\
\hline Factor de escala DLL & $550 \mathrm{e} 1$ \\
\hline
\end{tabular}

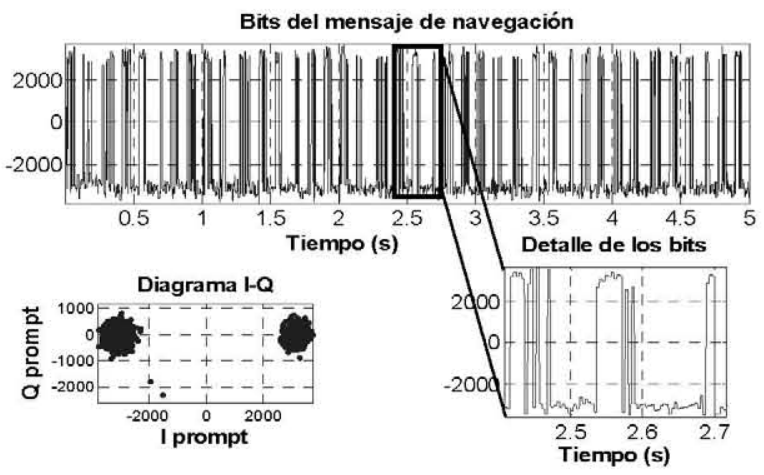

Figura 13. Bits de navegación extraídos de la captura de Giove-A realizada el 30-06-09 en Madrid con una elevación de $52^{\circ}$

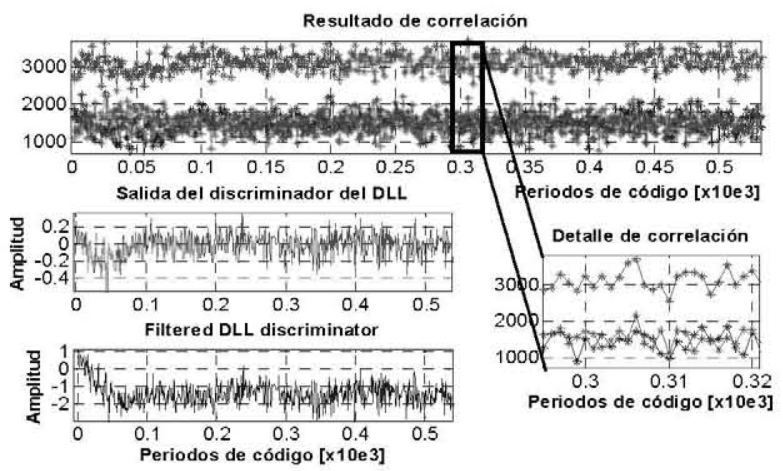

Figura 14. Resultados del DLL para la captura de Giove-A realizada el 30-0609 en Madrid con una elevación de $52^{\circ}$.

\section{Interfaz gráfica GAEDUNAV}

Cumpliendo con una parte importante de los objetivos y en la búsqueda de herramientas para ayudar al entendimiento de los sistemas de navegación global por satélite (GNSS), y en particular del sistema Galileo, se diseñó e implementó una sencilla interfaz gráfica que integrase todos los módulos software construidos, de una manera agradable a cualquier usuario. El resultado fue la herramienta GAEDUNAV (GAlileo EDUcational NAVigation tool). La interfaz implementada permite realizar las tareas básicas necesarias en un receptor GNSS, que son: adquirir y seguir la señal. Las señales utilizadas pueden provenir de capturas realizadas durante los pases del satélite, pero también se da la posibilidad de generar la señal de manera local y trabajar con ella, así como de visualizar la representación espectral de las señales capturadas.

Todas las señales pertenecen a la banda E1 de Galileo centrada en $1575.42 \mathrm{MHz}$. Los resultados generados por la interfaz son similares a los del programa original e ilustran de manera clara el funcionamiento de un receptor para GNSS Toda la interfaz se ha desarrollado en Matlab y está escrita en inglés para que pueda ser usada por un mayor número de personas.

Las entradas de datos ocupan casi todo el espacio de la interfaz ya que es la parte más importante porque con ellas se controla el desempeño del receptor. Están organizadas de manera simple e intuitiva, agrupándose por distintas categorías en función del módulo que controlen (

Figura 15.): Generales (a la izquierda y en rojo), adquisición (en la parte central superior y en verde), tracking (a la derecha y en azul), capturas (en la parte inferior y en amarillo) y generación (en la parte central inferior y en morado).

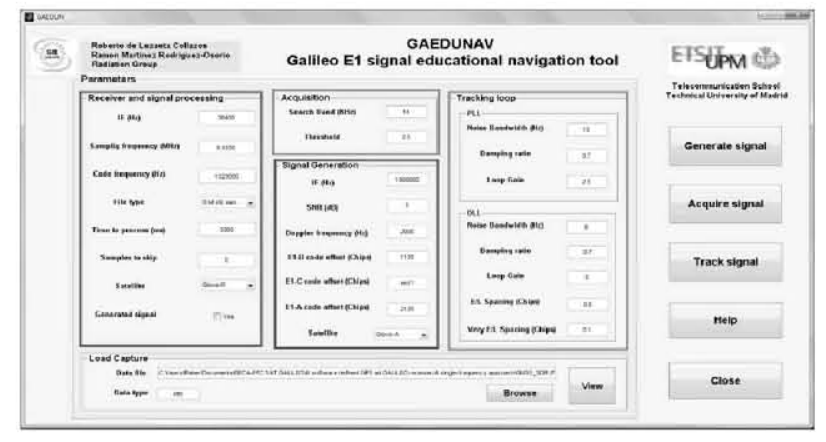

Figura 15. Interfaz gráfica GAEDUNAV

\section{Medidas}

\section{A. Planteamiento y escenarios}

Para probar el funcionamiento del sistema al completo se hicieron capturas de los dos satélites del sistema Galileo que están actualmente en el aire, el Giove-B y el Giove-A. Esto aporta un valor añadido al diseño ya que permite afirmar que el sistema funciona correctamente fuera del entorno controlado del laboratorio lo cual es muy interesante en la validación final de cualquier proyecto. Las capturas se realizaron en varios emplazamientos de la ETSIT-UPM en Madrid con distintas condiciones (visión directa, indoor y fuerte multitrayecto) representando escenarios típicos. Dichos escenarios se pueden ver en la Figural6. 


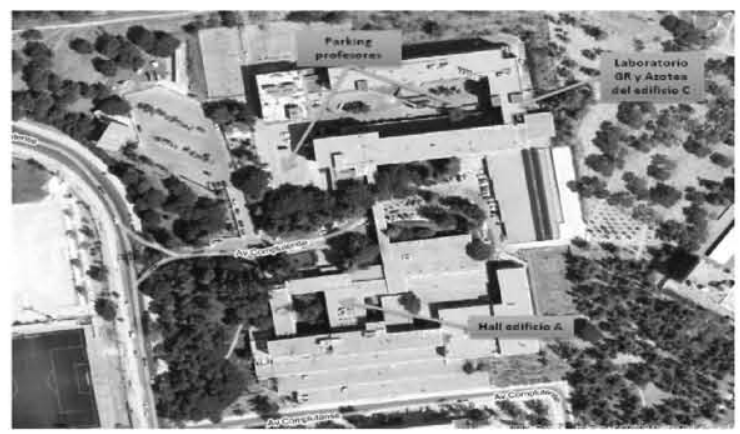

Figura16. Escenarios de medida

Los primeros escenarios en ser medidos fueron los de visión directa porque son los más benignos en cuanto a condiciones de recepción y de señal. Una vez se comprobó que el sistema funcionaba con este tipo de medidas se realizaron capturas en los otros dos escenarios.

\section{B. Hardware y setup}

Los equipos utilizados para las capturas se muestran en la Figura 17. Para realizar las capturas de utilizó el front-end monofrecuencia SiGE GN3S v2 comercializado por Sparkfun Electronics y una antena de diagrama semiesférico de $26 \mathrm{~dB}$ de ganancia, así como varias herramientas SW como el programa NOVA [8] para determinar las características de cada pase y programar el calendario de medidas, y los drivers del front-end. Todos los equipos demostraron ser eficientes y fiables (salvo el software del front-end que dio algunos problemas) durante toda la campaña de medidas, permitiendo la realización de varias series con resultados satisfactorios. La mayoría de las capturas fueron luego procesadas por el receptor software con resultados igualmente satisfactorios.
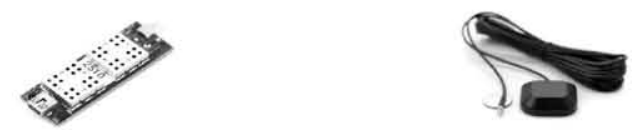

Figura 17. Equipos de medida. Front-end GNSS y antena

\section{Resultados}

La campaña de medidas dio como resultados una amplia serie de capturas en distintos escenarios que permitieron verificar la validez del receptor en la mayoría de los casos, salvo en aquellos con fuertes perturbaciones indoor. La mayoría de los espectros presentan similitudes en su forma y su amplitud, con pequeñas variaciones de uno a otro (Figura 18), debido a que en todos ellos están presentes las señales de navegación utilizadas en la banda L1 (GPS y Giove-A/B). Los resultados de adquisición de dicha captura se pueden ver en la Figura 19 .

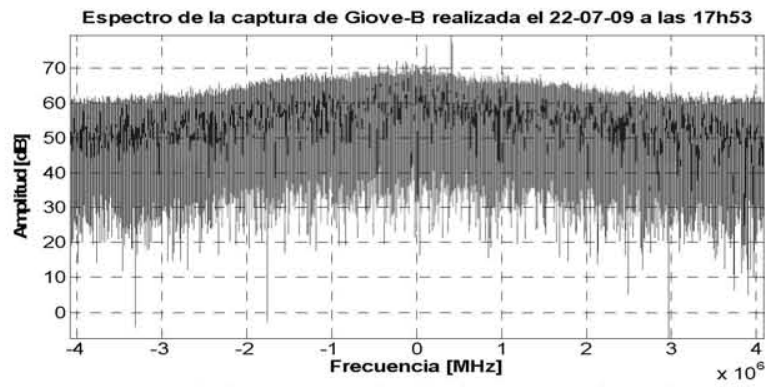

Figura 18. Espectro de la captura realizada el 22-07-09 a las 17 h53 con visión directa.

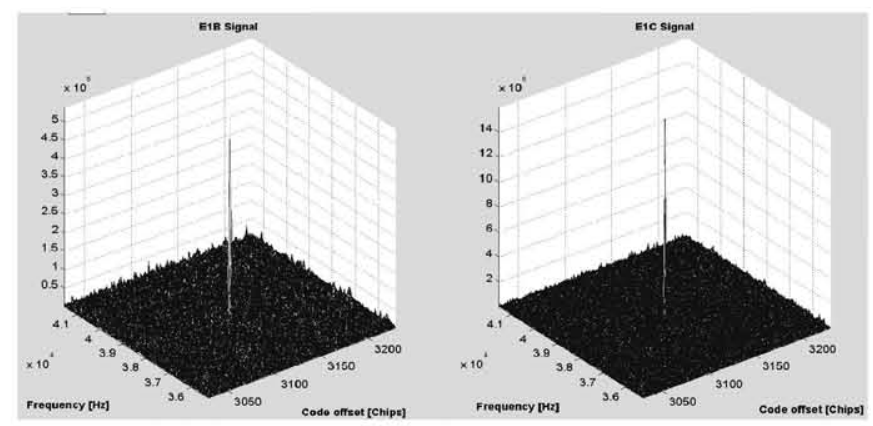

Figura 19. Matrices de correlación de la captura realizada el 22-07-09 a las 17 h53 con visión directa.

También se observó que en muchas de las capturas se presentaban señales interferentes muy potentes $y$ de procedencia indeterminada (Figura 18). Aunque no se logró determinar el origen de las interferencias situadas aproximadamente a $410 \mathrm{KHz}$ de la frecuencia central de emisión de la banda L1 (1575.42 MHz), se concluyó que no eran inherentes al equipo de medida porque de lo contrario aparecerían en capturas realizadas en distintas ubicaciones. Cabe pensar que dichas componentes provienen de un entorno cercano al laboratorio del GR de la ETSIT-UPM.

\section{CONCLUSIONES}

Los resultados de las pruebas demuestran que el concepto de receptor software es una opción interesante y versátil para GNSS como paso previo a su implementación en hardware o en software radio. Se piensa que el receptor diseñado también será capaz de adquirir y seguir a los próximos satélites (IOV) y a los satélites finales de la constelación con alguna pequeña adaptación (si es necesario).

Por otra parte, el esquema de adquisición desarrollado es adecuado para las señales E1B y E1C, dado que pueden ser adquiridas satisfactoriamente tanto si son generadas localmente en diferentes condiciones como si son capturas reales de señal. De igual manera la estructura usada para el seguimiento de la señal E1B permite obtener los datos de navegación de manera fiable con señales reales. Además, se cuenta con equipos para realizar capturas de señal del satélite Giove-A y Giove-B en diferentes entornos: visión directa, indoor, $\mathrm{y}$ con fuerte multitrayecto. Las pruebas muestran que para entornos con gran interferencia (indoor) no es posible adquirir la señal. 
La herramienta desarrollada es muy útil para demostrar de manera sencilla cómo funciona un receptor GNSS, cuáles son los procesos que se llevan a cabo y cuáles son los resultados arrojados en cada módulo. Por ello es recomendable su utilización en la docencia de sistema GNSS como herramienta de apoyo y prácticas.

Durante el desarrollo del proyecto surgieron ciertas incidencias, como por ejemplo el corte de emisión del GioveA desde el 7/7/2009 hasta el 7/9/2009, que obligaron a realizar cambios en el plan previsto. En este caso en particular, se adaptó el receptor para que fuese capaz de procesar también señales del Giove-B puesto que solo era posible capturar señales de dicho satélite.

\section{AGRADECIMIENTOS}

Los autores agradecen a la Cátedra ISDEFE de la ETSITUPM por La financiación y apoyo al proyecto. También se quiere agradecer a La Comisión Interministerial de Ciencia y Tecnología CICYT por La financiación al proyecto con referencia TEC2008-06736/TEC (CROCANTE).

\section{REFERENCIAS}

[1] Elliot D. Kaplan. "Understanding GPS: Principles and applications", $2^{\text {nd }}$ Edition, Artech House, 2006.

[2] "Giove - A + B Navigation Signal-in-Space Interface Control Document", Galileo Project Office, ESTEC 2008.

[3] Manuel Sierra Pérez, Belén Galocha Iragüen, José Luis Fernández Jambrina, Manuel Sierra Castañer. "Electrónica de Comunicaciones". Prentice Hall, 2007.

[4] Kai Borre, Dennis M. Akos, Nicolaj Bertelsen, Peter Rinder, Søren Holdt Jensen. "A Software-Defined GPS and Galileo Receiver. A Single-Frequency Approach”. Birkhäuser, 2007.

[5] Olivier Julien, "Design of Galileo L1F Receiver Tracking Loops", PhD Thesis, University of Calgary, Dpt. Of Geomatics Enineering. Canada. July 2005.

[6] Jinghui Wu, Andrew Dempster. "Galileo GIOVE-A Acquisition and Tracking Analysis with a New Unambiguous Discriminator". Proc. IGNSS Symposium 2007. The University of New South Wales, Sydney, Australia, Dec. 2007.

[7] Cheon Sig Sin, Jae Hyun Kim, Sanguk Lee, Jae Hoon Kim. "A Software Receiver Implementation for GPS LI and Galileo El Signal'. Proc. ASMS. Bologna, Italy, Aug. 2008.

[8] Programa de seguimiento de satélites (NOVA). www.nlsa.com

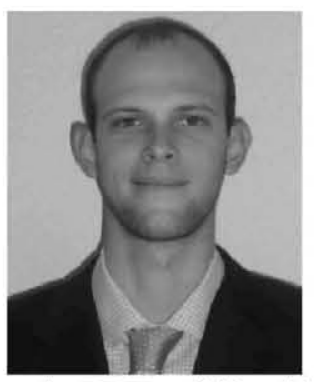

Roberto de Lezaeta Collazos nació en San Cristóbal, Venezuela, el 7 de Octubre de 1986. Obtuvo el título de Ingeniero de Telecomunicación con la especialidad de Comunicaciones en la E.T.S.I. de Telecomunicación de la Universidad Politécnica de Madrid (España) en 2009. Estudio durante un año en la Universidad Simón Bolivar (Caracas, Venezuela). Ha trabajado como Becario para la Cátedra ISDEFE de la ETSIT-UPM durante la realización de su Proyecto Fin de Carrera en receptores GNSS y ha realizado publicaciones en la XXIV edición del Simposium Nacional de la Unión Científica Internacional de Radio (URSI): R. de Lezaeta C., R. Martínez R.O. "Diseño de un receptor software radio para la recepción de señales de navegación del sistema Galileo". Santander, 16-18, Septiembre 2009. Así como en las XIX jornadas Telecom I+D: R. de Lezaeta C., R. Martínez R.O. "Diseño de un receptor software para señales del sistema Galileo". Madrid, 24-26, Noviembre 2009. Actualmente sus intereses van desde las comunicaciones por satélite a los sistemas GNSS con especial énfasis en receptores software radio y procesado de señales en los sistemas Galileo y GPS.'

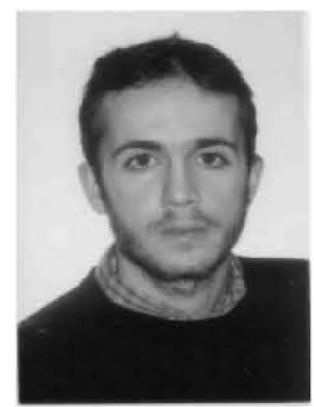

Ramón Martinez Rodriguez-Osorio nació en Madrid, España, el 25 de Septiembre de 1975. Obtuvo el título de Ingeniero de Telecomunicación en 1999 y en 2004 el título de Doctor en el Programa del Departamento de Señales, Sistemas y Radiocomunicaciones de la E.T.S. de Ingenieros de Telecomunicación de la Universidad Politécnica de Madrid. Desde 1999 desarrolla su actividad investigadora en la E.T.S. Ingenieros de Telecomunicación de la Universidad Politécnica de Madrid. Actualmente, es Profesor Titular de Universidad en el Departamento de Señales, Sistemas y Radiocomunicaciones de la UPM. Sus principales líneas de investigación son las antenas inteligentes y sistemas MIMO, las comunicaciones móviles por satélite y el procesado software radio. Ha participado activamente en diversos proyectos públicos europeos y nacionales, y ha sido el Investigador Principal de contratos con la industria. Ha publicado artículos en revistas internacionales de prestigio (IEEE, IET) y ha presentado contribuciones en Congresos Internacionales y Nacionales. También ha sido autor de capítulos en libros de simulación (Springer). E1 profesor Martínez Rodríguez-Osorio ha recibido Premios al Mejor Proyecto Fin de Carrera y su Tesis Doctoral por parte de del Colegio Oficial de Ingenieros de Telecomunicación y Telefónica Móviles. 